\title{
Die Promotionsarbeit des Joseph von Quarin als Spiegel des Standes der medizinischen Entomologie in der Mitte des 18. Jahrhunderts
}

Tilman Kiehne

\section{Summary}

Joseph von Quarin (1733-1814), court physician to the Emperor of Austria at the end of the 18th century, had studied medicine in Vienna and Freiburg im Breisgau. His Freiburg doctoral dissertation is still of great interest today. In a first chapter it defines the term «insect», in a second it describes diseases insects can cause, and a final chapter discusses medical uses of insects. Quarin tried to unite traditional knowledge with new findings. His brief work is a survey of the knowledge of medical entomology in his time.

\section{Zusammenfassung}

Joseph von Quarin (1733-1814), der Ende des 18. Jahrhunderts Leibarzt des Kaisers von Österreich war, verbrachte einen Teil seines Studiums an der Universität Freiburg im Breisgau. Seine dort 1751 verfasste Promotionsarbeit ist heute noch von grossem Interesse. In drei Kapiteln - von denen das erste den Terminus «Insekt» definiert, das zweite Krankheiten beschreibt, die Insekten verursachen können und das dritte Beispiele für den medizinischen Nutzen von Insekten gibt - versuchte Quarin das überlieferte Wissen mit neuen Erkenntnissen zu verbinden. So gibt seine Arbeit auf kleinstem Raum einen Überblick über den Stand der medizinischen Entomologie seiner Zeit.

Dr. Tilman Kiehne, Max-Rieple-Weg 6, D-79114 Freiburg/Br. 
Joseph Freiherr von Quarin ist keine der ganz grossen Gestalten der Medizingeschichte, aber auch kein unbeschriebenes Blatt: Leibarzt des Kaisers von Österreich, engagierter Professor und sechsmaliger Rektor an der Hochschule in Wien, gehörte er dort zu den besten Ärzten seiner Zeit.

Joseph Quarin wurde am 19. November 1733 in Wien als Sohn des Arztes Peter Quarin ${ }^{1}$ geboren. Ehrgeizig und hochbegabt nahm er in seiner Vaterstadt zunächst das Studium der Philosophie auf, das er $1748^{2}$ mit Erlangung der philosophischen Doktorwürde beendete. Im Anschluss daran widmete er sich dem Studium der Medizin ${ }^{3}$. Quarin arbeitete rasch und zielstrebig; 1750 wechselte er dann die Universität und immatrikulierte ${ }^{4}$ sich in Freiburg im Breisgau, um dort zu promovieren.

In kurzer Zeit verfertigte er seine Dissertation, die den Titel Entoma noxia et utilia physico-medice considerata trägt, und schrieb 34 Thesen, die er am 6. August 1751 vor der Fakultät verteidigte. Am 16. August 1751 wurde er zum Dr. med. promoviert ${ }^{5} .1752$ kehrte er nach Wien zurück und unterwarf sich dort dem actus repetitionis. Nachdem er diese nochmalige Überprüfung seines Wissens mit Bravour bestanden ${ }^{6}$ und damit die Erlaubnis erhalten hatte, zu praktizieren, nahm er in Wien seine Tätigkeit als Arzt auf.

Auf Anregung von Gerard van Swieten (1700-1772), dem Leibarzt der Kaiserin Maria Theresia, der den jungen Kollegen noch als Studenten erlebt hatte, begann Quarin ab 1754 zunächst Anatomie, später dann auch Arzneimittellehre und Therapie zu lehren. Daneben leitete er das Hospital der Barmherzigen Brüder ${ }^{7}$. 1758 ernannte ihn die Kaiserin auf van Swietens

1 Peter Quarin war - wie später sein Sohn - Doktor der Philosophie und Doktor der Medizin. Er übte das Amt des Professor praxeos an der Wiener Universität bis 1754 aus; dann liess ihn Gerard van Swieten im Rahmen seiner Reformen pensionieren (Lesky, S. 25, Anm. 57). Zu seinen Veröffentlichungen zählen die Disputatio medica de haemorrhoidibus (Wien 1732) sowie die Disputatio medico-physiologica de succo pancreatico (Wien 1741).

2 Welcker, S. 847. Auch für die damalige Zeit war die Promotion im Alter von 15 Jahren noch sehr früh.

3 Puschmann, S. 71, spricht davon, dass Quarin nur in Freiburg Medizin studiert habe. - Hier folge ich aber Welcker, S. 847, dessen Darstellung angesichts der kurzen Verweildauer und der schnellen Promotion an der Freiburger Universität nach nur zwei Semestern plausibler erscheint.

4 Matrikel 1750 Nr. 2 (Schaub, S. 607).

5 «6. Aug. 1751. Disputatio medica, defendente sine praeside Domino Guarini, sub cujus nomine prodiit dissertatio de insectis. - 16. Aug. Promotore D. Bader Prof. instit. medic. suprema laurea decorati sunt D. Guarini et tres alii.» (Schreiber, S. 194).

6 Üblicherweise wurde in geheimer Abstimmung über das Bestehen des Kandidaten entschieden; Quarin trat jedoch derart überzeugend auf, dass in seinem Fall diese Abstimmung entfiel und er per acclamationem zugelassen wurde (Welcker, S. 847).

7 Diese Tätigkeit, die er 28 Jahre lang ausübte, hatte er vom Vater übertragen bekommen (Welcker, S. 847). 
Vorschlag zum Regierungsrat und Referenten des Sanitätswesens an der niederösterreichischen Landesstelle ${ }^{8}$.

1777 gelang es Quarin, den Erzherzog Ferdinand zu heilen, der in Mailand erkrankt war, und dieser bestellte ihn zu seinem Leibarzt. Nach seiner Rückkehr aus Mailand wurde Quarin auch zum kaiserlichen Leibarzt erhoben und erhielt den Auftrag, die über Wien verstreuten Spitäler zu einem Allgemeinen Krankenhaus zusammenzufassen. Er löste diese Aufgabe in weniger als zwei Jahren, und 1784 wurde ihm die Oberdirektion dieses Krankenhauses übertragen ${ }^{9}$. Weitere vier Jahre später erhob ihn der Kaiser für seine Verdienste in den Freiherrnstand ${ }^{10}$.

Die Verdienste Quarins spiegelten sich nicht nur in seinen praktischen Erfolgen wider. Zeitlebens veröffentlichte er Werke, in denen er seine Erfahrungen anderen zugänglich zu machen suchte. So erschienen nach seiner Freiburger Dissertation schon im nächsten Jahr die Theses de salium differentiis et usu (Wien 1753). Es folgten ein Tentamen de cicuta (Wien 1761), der Methodus medendarum febrium (Wien 1772), der Methodus medendarum inflammationum (Wien 1774) und die Animadversiones practicae in diversos morbos (Wien 1786). Das letztgenannte Werk erschien wenig später unter dem Titel Praktische Bemerkungen über verschiedene Krankheiten (Wien 1787) auch auf deutsch ${ }^{11}$.

Nicht nur im medizinischen Bereich jedoch war Quarin aktiv: auch im Kampf gegen die Franzosen zeichnete er sich mehrfach aus: 1797, indem er dafür sorgte, dass in kurzer Zeit zwei Bataillone aus Studierenden aufgestellt werden konnten, die er teilweise aus eigenen Mitteln finanzierte, und 1805, als er bei der Besetzung Wiens die Plünderung der Sammlungen der Universität verhinderte, ja sogar erreichte, dass sie «den kräftigsten Schutz des Feindes genossen $»^{12}$.

Am 19. März 1814 schliesslich starb Joseph Freiherr von Quarin, ein «grosser Arzt, echter Patriot, wahrer Menschenfreund und unerschrockener Charakter» ${ }^{13}$.

8 Welcker, S. 847.

9 G(?), S. 697. - 1791 gab Quarin aus Ärger darüber, dass ihm zu wenig Geld für weitere Verbesserungen des Krankenhauses zur Verfügung gestellt werden sollte, diesen Posten wieder auf.

10 Nach Welcker (S. 848) eine Auszeichnung auch für Quarins Offenheit und Ehrlichkeit: Der Kaiser, der schon länger kränkelte, habe Quarin um eine Prognose gebeten, und dieser sich nicht gescheut, dem Kaiser zu erklären, dass er nur noch eine kurze Frist voraussehe. Am nächsten Tag habe ihm dann Joseph die Ernennung zum Freiherrn und ein grosszügiges Geldgeschenk übersandt.

11 Auch andere seiner Werke wurden unter anderem ins Deutsche, Italienische, Englische und Französische übersetzt und erreichten so einen grossen Leserkreis.

12 Welcker, S. 849.

13 Welcker, S. 850. 
Die medizinische Wissenschaft hatte sich im 18. Jahrhundert, «nach eigenem Verständnis, aus den Fesseln der Autoritätsgläubigkeit befreit, wollte nicht mehr nach dem Ansehen der Person, sondern nach dem in der Sache, in Vernunft und Erfahrung gegründeten Urteil verfahren ${ }^{14}$.» Die Grundsätze der Medizin, die Hermann Boerhaave in Leiden aufstellte, begannen schon zu dessen Lebzeiten, den Kontinent zu durchdringen. In Wien legte van Swieten, Boerhaave folgend, in Theorie und Praxis grössten Wert auf die Beobachtung; besonders wichtig wurde die Verlaufsbeobachtung der Krankheiten am Krankenbett durch die Visite ${ }^{15}$. Joseph Quarin wiederum hatte bei van Swieten in Wien Vorlesungen gehört, und sein medizinisches Denken war von diesem stark beeinflusst. Die Verhältnisse der Wiener und nicht die der Freiburger medizinischen Fakultät waren es, die Quarin prägten; und gewiss ist es auch kein Zufall, dass sein Promotor Franz Josef Lambert Bader war, der selbst sieben Jahre lang in Wien bei van Swieten gelernt hatte ${ }^{16}$.

Als Quarin nach Freiburg kam, war die dortige medizinische Fakultät klein und die Universität Freiburg noch «dem Gesamteindruck nach ... unbedeutend, provinziell und eifersüchtig darauf bedacht, ihre Privilegien zu hüten - alles in allem ein Stück noch lebendigen Mittelalters, mit manchem barocken Gewand überkleidet ${ }^{17}$.» An diese im Vergleich zu Wien in vielerlei Hinsicht rückständige Universität wechselte Quarin gewiss nicht, weil er sich neue Erkenntnisse erhoffte. Was an Freiburg für ihn reizvoll war, war ledig-

14 Toellner, S. 221.

15 Lesky weist darauf hin, dass der Unterricht am Krankenbett in Wien keine prinzipielle Neuerung darstellte. «Der Krankenbett-Unterricht hat eine lange, wenn auch nicht ungebrochene Tradition, die bis 1389 zurückgeht» (Lesky, S. 25, Anm. 57). Jedoch habe van Swieten vor allem durch zwei Anordnungen eine wesentliche Verbesserung dieses bestehenden Unterrichts erreicht: Erstens habe Anton de Haen - der Nachfolger Peter Quarins im Amt des Professor praxeos - sich die Kranken, die er vorstellen wollte, nun an allen Wiener Krankenhäusern auswählen können, und zweitens habe der Unterricht am Krankenbett nach der Reform täglich statt wie vorher in unregelmässigen Anständen stattgefunden (a.a.O., S. 25/26).

16 Seidler, S. 54.

17 Zeeden S. 49. - Der Wandel dieses Zustands stand allerdings unmittelbar bevor. 1748 hatte Gerard van Swieten auf Geheiss der Kaiserin Maria Theresia in Wien mit einer Reform der medizinischen Ausbildung begonnen, die derart erfolgreich war, dass sie als Modell für alle medizinischen Fakultäten in Österreich gelten sollte. Diese umfasste sowohl eine Umstrukturierung der bisherigen Universitätsverfassung als auch einen Bruch mit den bisherigen Lehrmethoden: Mehr als bisher sollte das «vorzeigbare Objekt, sei es der menschlichen Leiche im Anatomischen Theater, der Pflanze im Botanischen Garten, der chemischen Substanz im Laboratorium oder des kranken Menschen am Krankenbett» (Lesky, S. 24) im Vordergrund stehen und die Buchgelehrsamkeit zurückgedrängt werden;zudem wurden die antiken Autoren ersetzt von modernen wie Hermann Boerhaave, Carl Linné oder Jakob Winslow (Seidler, S. 63). 
lich die Möglichkeit, den erstrebten Doktortitel schneller und billiger zu erlangen, als es in Wien ${ }^{18}$ möglich war ${ }^{19}$.

Grundsätzlich führten zur Zeit Quarins zwei Wege zur Promotion: Zum einen konnte eine Dissertation - geschrieben vom Prüfling selbst oder, häufiger, vom Doktorvater - vor der Fakultät vorgestellt und verteidigt werden, zum zweiten konnte der Prüfling Thesen vorlegen, anhand derer er zeigen konnte, dass er ärztlich zu denken und zu handeln imstande war.

Joseph Quarin war sicherlich ein überdurchschnittlicher Student. Er ging - und das hebt ihn aus der Masse hervor - beide Wege: Zum einen verfasste er selbst ${ }^{20}$ die hier besprochene Arbeit über die Insekten, zum anderen legte er darüber hinaus noch 34 Thesen $^{21}$ vor, die als Diskussionsgrundlage bei der Prüfung dienen konnten.

\section{III}

Die Beobachtung, Beschreibung und Klassifizierung der Insekten ${ }^{22}$ ist lange Zeit von untergeordnetem Interesse gewesen, obwohl schon seit dem Altertum durchaus ein Interesse an der Insektenkunde vorhanden war. Bis zur Erfindung des Mikroskops fehlte es jedoch an geeigneten Apparaturen zur Erforschung der dem blossen Auge vielfach kaum zugänglichen Strukturen. So fanden zunächst im wesentlichen diejenigen Kleinlebewesen Beachtung, die dadurch auffielen, dass sie dem Menschen schadeten, wie Läuse, Flöhe,

Die Reform hatte selbstverständlich auch Auswirkungen auf die - damals noch zu Österreich gehörende - Freiburger Fakultät: Ein botanischer Lehrgarten wurde geschaffen, ein neues Universitätsgebäude errichtet und der Unterricht am Krankenbett wurde neu geregelt. Auch waren schon vor Quarins Studienzeit die ersten Professoren an die Freiburger Universität gekommen, die in Wien die Reformen kennengelernt hatten, wie zum Beispiel Franz Josef Lambert Bader (1723-1773). Insgesamt jedoch sperrten sich die Verantwortlichen bei Senat und Fakultät noch gegen die geplanten Reformen, und erst 1768 wurde nach einem grossen Eklat - der Regierungskommissar Hermann von Greiffenegg liess 1767 die Tore der Universität aufbrechen, um sich Zugang zu Archiv, Kanzlei, Registratur und Kasse zu verschaffen - eine neue Universitätsverfassung geschaffen (Zeeden, S. 61).

18 In Wien wurde der medizinische Doktorgrad nur alle sechs Jahre in einem grossen Festakt im Stefansdom verliehen und die Feier war mit 1000 Gulden, die die Promovenden zu zahlen hatten, entsprechend teuer (Lesky, S. 21).

19 So zumindestens Welcker, S. 847. Auch der Lebenslauf Quarins - nachdem er sein Ziel erreicht hatte, zog es ihn sehr bald zurück nach Wien - spricht für diese These.

20 Zumindestens «aller Wahrscheinlichkeit nach» (Nauck, S. 68).

21 Die Thesen, die der Student Quarin verteidigte, zeugen ebenfalls von dessen umfassenden Anspruch. In der gedruckten Fassung der Dissertation Quarins sind sie als Positiones ex universa medicina im Anhang enthalten; da sie jedoch keinen direkten Bezug zum Thema der Dissertation haben, bleiben sie aus dieser Arbeit ausgeklammert.

22 Zum Terminus «Insekt» vgl. Anm. 39. 
Bienen, Wespen, Hornissen o.ä. ${ }^{23}$, und durch deren nähere Betrachtung man sich daher einen direkten Nutzen für das alltägliche Leben - nämlich das Abwenden von Krankheit und Gefahr - erhoffen konnte.

Erst Aristoteles (384-322 v.Chr.) wagte den Versuch einer allgemeinen Systematisierung der Insektenwelt. Er tat dies so gründlich, dass sein umfangreiches Material noch lange Zeit die einzige Quelle und Grundlage aller weiteren Beschäftigung mit der Entomologie blieb. Aristoteles, der auf vielen Gebieten bahnbrechend war, kann daher mit Fug und Recht auch als der Begründer der allgemeinen Entomologie und der Entomologie als Wissenschaft angesehen werden. Ein weiterer Grieche, Dioscorides, bereitete in der Mitte des 1. Jahrhunderts nach Christus die Grundlagen einer entomologischen Pharmakologie.

Die Römer erweiterten das von den Griechen überlieferte und erworbene Wissen nur um wenige Anmerkungen, die Plinius (23-79) dem neunten Buch seiner Historia naturalis über die Insekten eingliederte ${ }^{24}$. Ab dem 2. Jahrhundert setzte sich dann langsam der Physiologus, ein Werk unbekannter Verfasser, als das Buch durch, das die Vorstellungen der Menschen über die Insekten entscheidend prägte. Dieser markierte einen Rückschritt gegenüber dem Werk des Aristoteles, da er weniger Wert auf Naturbeobachtung als auf christliche Natursymbolik legte: Die Eigenschaften der dargestellten - teils wirklichen, teils mythischen - Tiere dienten zur Darstellung christlichen Glaubensguts ${ }^{25}$.

Im 13. Jahrhundert lebte das Interesse an wissenschaftlicher Entomologie wieder auf. Zwei Autoren waren hier von besonderer Bedeutung, obwohl sie im wesentlichen die Lehren des Aristoteles wiedererweckten: Albertus Magnus (1193-1280) mit seinen 26 Bücher De animalibus, und dessen Schüler Thomas von Cantimpré (1201-zwischen 1263 und 1293) mit seinem 19 Bücher umfassendes Liber de natura rerum, dessen 9. Buch sich mit den «Würmern» befasste, unter die er auch Amphibien und Insekten eingliederte. Besonders das Liber de natura rerum fand aufgrund seiner raschen Übersetzung ${ }^{26}$ ins Deutsche weite Verbreitung.

Der weitere Weg der Entomologie führt über den Hortus Sanitatis des Johannes Wonneke von Caub (1480), der die ersten - wenn auch noch groben

23 In diesem Zusammenhang sei auch an die zehn biblischen Plagen erinnert, von denen die dritte (Stechmücken), die vierte (Ungeziefer) und die achte (Heuschrecken) unmittelbar durch Insekten verursacht wurden (2. Mose, 8-10).

24 Für eine detaillierte Darstellung der Entwicklung der Entomologie im Altertum sei hier verwiesen auf Morge, S. 38-49 (Griechenland) und S. 49-54 (Rom).

$25 \mathrm{Jahn/Löther/Senglaub,} \mathrm{S.} 94$.

26 Von Konrad von Megenburg (1309-1374) als Buch der Natur. - Der Übersetzer nahm übrigens an, Albertus Magnus sei der Verfasser des Werks gewesen (Morge, S. 69). 
und unvollständigen - Abbildungen einführte, und die De Animalibus Insectis libri VII (1602) des Ulysse Aldrovandi (1522-1605) zu dem Apiarium des Frederico Cesi (1625), dem «oldest book based on microscopical studies» ${ }^{27}$.

Mit der Erfindung des Mikroskops ergaben sich für die an Insekten interessierten Forscher völlig neue Möglichkeiten, und so wurde im 17. Jahrhundert die Entomologie entscheidend vorangetrieben. Marcello Malpighi (1628-1694) veröffentlichte mehrere Monographien, die sich mit Themen aus der Insektenkunde befassten. Antony van Leeuwenhoek (1632-1723), der seine Mikroskope «on everything which came near him $»^{28}$ richtete, veröffentlichte von 1695 bis 1722 vier Bände Arcana Naturae Detecta Ope Microscopiorum. Und von 1734 bis 1742 erschienen dann die sechs Bände umfassenden Mémoires pour servir à l'histoire des insectes von René Antoine Réaumur.

Zunehmend wurden jetzt auch Versuche unternommen, die Insekten in Systeme zu klassifizieren. Jan Swammerdam (1639-1680) legte 1669 eine Historia Insectorum Generalis vor, in der die Insekten aufgrund der Unterschiede in ihrer Entwicklung klassifiziert wurden ${ }^{29}$. Antonio Vallisnieri (1661-1730) schuf eine neue Einteilung der Insekten nach ihrem bevorzugten Aufenthaltsmedium. Eine weitere Einteilung der Insekten - nach morphologischen und biologischen Kriterien - lieferte John Ray (1628-1705) in seiner Historia insectorum. 1735 schliesslich erschien das Systema Naturae von Carl von Linné, in der die Insekten primär nach der Beschaffenheit ihrer Flügel klassifiziert wurden ${ }^{30}$.

\section{IV}

Joseph Quarin formuliert im Vorwort seiner Promotionsarbeit als Anspruch, er wolle einen Bogen zu schlagen von der sapiens vetustas zur medendi scientia. Beim Lesen wird jedoch schnell klar, dass die sapiens vetustas nur eine untergeordnete Rolle bei Quarins Überlegungen spielt; bestimmend sind ganz klar zeitgenössische Autoren. So werden in Quarins Promotionsarbeit auf neunundzwanzig Seiten die Grundzüge des entomologischen Wissens in der Mitte des 18. Jahrhunderts dargestellt, und das macht seine Arbeit für den heutigen Leser noch immer interessant.

27 Beier, S. 88.

28 Tuxen, S. 97.

29 Swammerdams Hauptwerk, die Biblia naturae (1737/38), wurde - u.a. durch Hermann Boerhaave - erst posthum aus weiteren seiner Schriften zusammengestellt.

$30 \mathrm{Zu}$ den verschiedenen Systematisierungsversuchen vgl. den Abschnitt «Systematizing» bei Tuxen, S. 105-113. 
Das erste Kapitel, Über den Ursprung und die Natur der Insekten ${ }^{31}$, ist noch stark von Aristoteles beeinflusst. So nimmt Quarin bei der Definition des Gegenstands seiner Untersuchung ausdrücklich auf dessen Historia animalium Bezug.

Es handele sich zunächst bei allen kleinen Tierchen um Insekten, deren Körperbau so beschaffen sei, dass er durch Einschnürungen in zwei oder drei Teile gegliedert sei, die quasi nur durch einen Faden verbunden seien, also «Spinnen, Ameisen, Wespen, Bienen, ... usw.» ${ }^{32}$. Daneben jedoch treffe die Bezeichnung Insekten in einem weitergefassten Sinn auch auf andere Tiere zu, deren Körper nicht ganz so stark eingeschnitten sei, die aber dennoch «aus mehreren untereinander verbundenen Ringen $»^{33}$ erschienen, so z.B. «Spulwürmer, Blutegel, Bandwürmer, Askariden und andere Würmer, ob nun mit oder ohne Beine» ${ }^{34}$. Aber auch jene Tiere, deren Körper mit beweglichen, untereinander verbundenen Schuppen, Schalen oder Knochen bedeckt seien, seien unter die Insekten zu zählen, also «Fliegen, Giftkäfer, Maikäfer, Krebse, Skorpione, usw.» ${ }^{35}$. Und schliesslich würden von manchen hochgelehrten Männern ${ }^{36}$ auch noch solche Tiere zur Gattung der Insekten gerechnet, die in allen Körperteilen auch dann weiterlebten, wenn sie durchschnitten würden: «Regenwürmer, Bandwürmer, Schlangen, usw.» ${ }^{37}$. Weitere Tiere, auf die dieses Merkmal zutreffe, seien zu finden unter Seesternen usw. ${ }^{38,39}$.

31 De insectorum ortu et natura ( $\S$ I-XVIII). [Alle Quellenangaben der Form «§ ...» beziehen sich hier wie im folgenden Text auf die Promotionsarbeit Joseph Quarins.]

32 «araneae, formicae, vespae, apes, ... \&c.» (§ II).

33 «ex pluribus annulis ... inter se connexis» (\$ III).

34 «lumbrici, hirudines, taeniae, ascarides, aliique vermes seu pedibus instructi seu iisdem destituti» (\$ III).

35 «muscae, cantharides, scarabaei majales, cancri, scorpiones, \&c.» (§ IV).

36 Hier führt Quarin Friedrich Hoffmann (1660-1742) an. Er weist zugleich einschränkend darauf hin, dass ab diesem Punkt von «Insekten» nur noch in erweiterten Sinn («licet non nisi sub latiori quodam titulo») gesprochen werden kann. Quarin selbst hält es, wie sich im weiteren Verlauf der Dissertation anhand der Belegstellen zeigt, mehr mit Carl von Linné, der schon in der ersten Auflage seines Systema Naturae die Würmer nicht mehr den Insekten zugerechnet hatte.

37 «lumbrici terrestres, toeniae, serpentes \&c.» $(\S \mathrm{V})$.

38 § VI.

39 Der Begriff «Insekt» ist heute nur noch Bezeichnung für einen kleinen Ausschnitt aus der Tierwelt, nämlich die Hexapoda, die gemeinsam mit den Spinnentieren (Arachnidae) und den Krebsen (Crustaceae) unter dem Stamm der Gliedertiere (Articulata) zu finden sind. Die Würmer haben mit den Insekten nach der heutigen Klassifizierung nur noch die Stammreihe (Protostomia) gemeinsam, in der sie eigene Stämme bilden. (Z.B. die Plattwürmer (Plathelminthen), Schnurwürmer (Nemertini) und Rund- oder Schlauchwürmer (Aschelminthen).

Die Schlangen wiederum werden heute ganz anders eingeordnet, nämlich in der Klasse der Reptilia bei den Wirbeltieren. - Zu speziellen Fragen der Systematik vgl. Gessner; einen kurzen Überblick liefert z.B. Vogel/Angermann, S. 224-265. 
Ebenfalls zu «Ursprung und Natur der Insekten» gehört das Thema «Fortpflanzung», dem Quarin den zweiten Teil des ersten Kapitels widmet: Ausgehend von der Regeneration der beiden Teile eines zerschnittenen Regenwurms, die er ausführlich beschreibt, stellt er zunächst fest: Niemand habe bisher befriedigend erklären können, wie solches möglich sei. Ebenso bewunderungswürdig wie diese Fähigkeit von Pflanzen, Insekten und anderen niederen Tieren sei jedoch, dass «Pflanzen und Tiere durch einige bislang noch nicht gut bekannte Arten der Fortpflanzung $»^{40}$ verbunden seien.

Und jetzt geht er über Aristoteles hinaus. Früher, so schreibt er, habe man mit Aristoteles an drei Fortpflanzungsarten geglaubt: «entweder aus Eiern, aus Fäulnis oder von Eltern» ${ }^{41}$. Dies habe aber nur in der Zeit vor dem Mikroskop behauptet werden können und sei inzwischen widerlegt. Quarin folgt anderen Autoritäten; er nennt Vallisnieri, Leeuwenhoek, Swammerdam, Redi und Malpighi. Deren Arbeiten ${ }^{42}$, so schreibt er, hätten bewiesen, dass die Befruchtung eines weiblichen Eis mit männlichem Samen ein Universalgesetz sei, die für eierlegende Lebewesen genauso Geltung habe wie für solche, «die einen Föten bis zum vollendeten Reifungszustand und dem Ende der Reifungszeit auf einem Nährboden im Körper zurückhielten ${ }^{43}$.

Gewiss kämen aus manchem, was in Fäulnis übergegangen sei, verschiedenste Tiere, aber nicht darum, weil sie dort entstünden, sondern, weil dort «aufgrund des natürlichen Instinkts, mit dem alle Tiere für ihre Fortpflanzung und Erhaltung sorgten ${ }^{44}$ die Insektenmütter ihre Eier in einer Umgebung ablegten, die für die Entwicklung der Jungen geeignet sei. Dies beweise ein Versuch: «Man lege zwei Pfund frisches Fleisch in zwei unterschiedliche Gefässe, eine Portion in ein Gefäss, das durch einen Stopfen verschlossen ist, und eine in ein offenes; so dass jedoch in beide Gefässe die Luft frei ein- und austreten kann: so wird die zweite Portion Fleisch Würmer und Mücken hervorbringen, da die Mücken frei ihre Eier darin ablegen können; die andere aber wird zwar genauso verrotten, aber weder Würmer noch Mücken erzeugen $»^{45}$. Absurd sei die Vorstellung, die manche äusserten, das Ganze

40 «plantae \& animalia quaedam plures, \& necdum bene cognitos, propagandi modos» (§ VII). 41 «aut ex ovo, aut ex putredine, aut ex parentibus» (§ IX).

42 Es war Francesco Redi (1626-1698), der die These aufstellte, dass alles Leben aus Eiern hervorgehe.

43 «qui foetum usque ad perfectae maturationis gradum \& tempus in matrice intra corpus retentum» $(\S \mathrm{XIV}-\mathrm{XV})$.

44 «instinctu naturali, quo animalia omnia in sui propagationem ac conservationem feruntur» $(\S \mathrm{XI})$.

45 «reponantur duae librae carnis recentis in vitra duo distincta, una in vitrum syndone clausum, in apertum altera; ita ut in utrumque aer libere ire ac redire possit: ultima haec vermes muscasque progenerabit, cum muscae libere in illam ova sua deponere queant; altera licet aeque corrumpatur, nec vermes nec muscas dabit» (§ XII). 
geschehe durch das zufällige Zusammentreffen von kleinen Partikeln; dies sei ähnlich absurd wie, wenn jemand behaupten wolle, die «Ilias Homers sei aus dem zufälligen Zusammentreffen von Buchstaben entstanden ${ }^{46}$.

Der Schluss des ersten Kapitels unterstreicht das Anliegen, das Quarin im Vorwort formulierte, nämlich, eine Brücke von den alten Autoren zu den neuen zu schlagen:Wenn manche Autoren früher zu beobachten gemeint hätten, dass verschiedene Tiere demselben Ei entstammen könnten, so könne man ihnen das nicht zum Vorwurf machen. Das habe allein daran gelegen, «dass diese Insekten ... nicht durch ein ewiges und beständiges Naturgesetz in derselben Form heranwachsen und auftreten ${ }^{47}$. Manche Insekten erhielten erst mehr oder weniger lang nach dem Schlüpfen ihre endgültige Gestalt. Und diese «transmutatio», diese vollständige Veränderung, sei es gewesen, «die bisher die Vorstellungsgabe vieler täuschte» ${ }^{48}$.

Das zweite Kapitel, Über den Schaden, der von Insekten verursacht wird ${ }^{49}$, befasst sich mit der Schädigung des Körpers durch «Insekten» im Sinne der obigen Definition.Zunächst stellt Quarin fest, dass «die Lebenskraft, die den Körper ernährt, ihn durch dieselben Kräfte auch zerstört» ${ }^{50}$. Daraus folgt, dass es für die Insekten prinzipiell drei Eintrittspforten gibt, nämlich die, durch die der Körper auch das aufnimmt, was er benötigt, um sich am Leben zu erhalten: «Dieselben [= die Insekten] werden entweder mit Essen und Trinken hinuntergeschluckt, mit der Luft ... während der Einatmung eingesogen, oder kommen schliesslich mit der Hülle unseres Körpers in Kontakt» ${ }^{51}$. Diese drei möglichen Eintrittspforten belegt Quarin in der Folge anhand von Beispielen.

Bei der Entstehung der Krankheiten spiele die eingeatmete Luft ${ }^{52}$ «aufgrund der unermesslichen Zahl kleinster Insekten, die in ihr umherflögen», eine so grosse Rolle, dass Hippokrates diese gar als Verursacher aller Krankheiten $^{53}$ bezeichnet habe. Die Luft der Sümpfe sei zwar von manchen Autoren als besonders schlimm hervorgehoben worden, aber Insekten fänden sich überall und liessen sich dann auf geeigneten Nährböden ${ }^{54}$ nieder, um sich

46 «Homeri Iliades ex fortuito literarum concursu coaluisse» (§ XIII).

47 «quod illa insecta ... non perenni \& constanti lege naturae sub eadem forma crescant \& appareant» (§ XVI).

48 «quae adeo multorum ingenia torsit» ( $\$$ XVII).

49 De insectorum noxis ( $\$$ XIX-XLIII).

50 «eadem vis vitae, quae idem [=corpus] nutrit, iisdem viribus quoque illud destruat» $(\S X X)$.

51 «Eadem [=insecta] nempe vel cum cibo ac potu \&c. deglutiuntur; vel cum aere ... in inspiratione adducuntur; vel denique ... corporis nostri peripheriae applicantur» ( $\$ \mathrm{XXI})$.

52 «incredibili copia minimorum insectorum, quae in eo mire circumvolitant» (\$XXII).

53 «fabrum omnium morborum» (a.a.O.).

54 Sogar die Nase kann einen - wenn auch ungewöhnlichen - Nährboden darstellen: «Der berühmte van Swieten berichtete in seinen Vorlesungen, es habe in den Niederlanden eine Frau gelebt, die den Geruch der Blumen so sehr liebte, dass sie im Frühjahr ständig Blumen, 
dort zu vermehren, wie «ich bei unserem berühmten Professor der praktischen Medizin mit einem äusserst genauen Mikroskop untersuchen durfte» ${ }^{55}$.

Die Abschnitte XXX bis XXXIV befassen sich mit Wurmerkrankungen als Beispiel für Erkrankungen, die aufgrund der Nahrung entstanden sind. Die Symptome der Wurmerkrankung seien so vielgestaltig, dass es «nach Baglivius keine noch so fremdartigen oder bedrohlichen Erscheinungen gibt, die Würmer nicht hervorrufen können ${ }^{56}$, und daher verwundere es nicht, dass Ungebildete die Wurmerkrankung oft auf Verwünschungen oder Dämonen zurückführten. Drei Arten von Würmern verursachten die meisten Erkrankungen der menschlichen Eingeweide:Helminthen,Tänien und Askariden; allerdings seien diese nicht spezifisch für den Menschen, und könnten auch andere Körperteile als den Darm befallen, wie z.B. die Lungen, Nase, Zahnfleisch oder Zähne. Als Autoren, die darüber ausführlich berichtet hätten, gibt Quarin hier seinen Wiener Lehrer van Swieten und den ehemaligen Freiburger Stadtarzt Johannes Schenck von Grafenberg (1531-1598) an.

Und schliesslich wendet er sich den Krankheiten der Haut zu und wählt hier als Beispiel «die Scabies, die durch Kontakt oder Ansteckung erworben wird, die innerhalb der Cutis und der Epidermis verweilt und unerträglichen Juckreiz und Reizung hervorruft ${ }^{57}$; eine Krankheit, deren Ursprung zwar umstritten sei, aber aufgrund der erfolgreichen Behandlung mit «Quecksilber, das Würmer abtötet $\aleph^{58}$ und anderer Indizien auch auf Insekten zurückgeführt werden müsse.

Die nächsten beiden Abschnitte widmen sich der Symptomatik nach Stichen bzw. Bissen von Schlangen, Bienen, Wespen, Spinnen und Skorpionen. Worauf diese beruht und warum sie je nach dem Verursacher so unterschiedlich ausgeprägt ist, kann Quarin nur vermuten: «Vielleicht erbrechen auch einige Insekten irgendeinen giftigen Saft in unsere Haut, der diese dann auf verschiedene Weise erkranken lässt» ${ }^{59}$.

die sich durch ihren Geruch auszeichneten, zur Nase führte und die Nase hineinsteckte.... In einem fürchterlichen Schauspiel entsprangen ihrer Nase einige tausend Insekten verschiedener Art» («Illustrissimus van Swieten in collegiis suis retulit, fuisse in Belgio foeminam, quae odorem florum adeo adamavit, ut verno tempore continuo flores quosdam grato odore se commendantes naribus admoveret, imo iis intruderet. ... Horrendo spectaculo aliquot millia insectorum diversae speciei ... e naribus excrevit») ( $\$$ XXVI).

55 «mihi apud Clarissimum nostrum Professorem Praxeos acuratissimo microscopio examinare licuit» (§XXV). - Bei dem im Text nicht namentlich genannten Professor praxeos handelt es sich um Franz Josef Lambert Bader, den Promotor Quarins (Seidler, S. 502).

56 «teste Baglivio nullum tam peregrinum tamque Daimoniakon est symptomata quod vermes excitare non possint» ( $§ X X X I)$.

57 «Scabiem illam, quae per attactum aut contagium acquiritur», die «intra cutem \& epidermidem haeret, mox intolerabilis pruritus \& irritatio exoritur.» (§ XXXV)

58 «mercurio vermes enecante» (a.a.O.)

59 «Forte etiam plura insecta venenatum quendam humorem in cutem nostram evomunt, qui varie dein hanc afficit» (§XXXVII). 
Der Rest von Kapitel II widmet sich den epidemisch und endemisch auftretenden von Insekten verursachten Krankheiten. Für die Epidemien beschreibt Quarin einen Zusammenhang zwischen dem Auftreten von gehäuften Erkrankungsfällen und einer erhöhten Zahl von Insekten in der Luft: In Ungarn und anderswo beispielsweise «nehmen die Einwohner als sicheren Beweis einer bevorstehenden Pest die ungeheuere Zahl von Heuschrecken ${ }^{60}$. Dennoch glaube er nicht, dass jedes Auftreten von Pest oder Fieberepidemien immer durch Insekten verursacht sei. Er wolle nur festhalten, «dass in der Luft Insekten und Insekteneier vorkommen, die, wenn sie auf verschiedenen Wegen und verschiedene Weise in oder an unseren Körper gelangt sind, ... viele, unterschiedliche und schwere Erkrankungen, nicht selten auch Epidemien, hervorrufen können und oft auch hervorrufen» ${ }^{61}$.

Für örtliche Unterschiede in Infektiosität und Schweregrad der Erkrankung hingegen kann Quarin keine Erklärung liefern. In diesem Fall hält er lediglich Beispiele fest wie zum Beispiel: «Der Stich der Tarantel ist nur in der italienischen Landschaft Apulien, und dort am meisten in der Gegend um Tarent, woher sie auch ihren Namen hat, schädlich und giftig; in allen anderen Gebieten schadet er entweder nur selten, oder viel weniger, oder gar nicht ${ }^{62}$. Und als Quintessenz des zweiten Kapitels bleibt der lapidare Satz «Die meisten Erkrankungen rühren also von Insekten her» ${ }^{63}$.

Den Nutzen der Insekten darzustellen, fällt Quarin deutlich schwerer, als deren Schädlichkeit. So beginnt er das dritte Kapitel, Über die Nützlichkeit der Insekten ${ }^{64}$, mit einer allgemein-theologischen Erwägung: nämlich, dass «der Schöpfer aller Dinge selbst, als er ansah, was er geschaffen hatte, alles für sehr gut befand» ${ }^{65}$. Und wenigstens einen Nutzen der Insekten könne keiner bestreiten, nämlich, «dass sie als Mittel der Strafe Gottes wirken können und oft gewirkt haben» ${ }^{66}$.

Jedoch besässen einige Insekten durchaus spezifische heilsame Kräfte: «Sie bieten uns Medikamente» ${ }^{67}$, ja, «tatsächlich erhalten wir aus ihnen auch

60 «incolae fere certum indicium futurae pestis ex ingente locustarum numero hauriunt» ( $\mathrm{XXXIX).}$

61 «esse in aere insecta et insectorum ova, quae per varias vias \& modos corpori nostro ingesta vel applicata ... multos, varios, magnosque morbos, atque etiam non raro epidemicos producere possint \& saepe produxerint» ( $\$ \mathrm{XL})$.

62 «Tarantulae ictus in sola Apulia Italiae regione, atque vel maxime in vicinia Tarenti, unde nomen habet, noxius \& venenatus est: in reliquis regionibus aut rarissime aut longe minus, vel omnino non nocet.» (\$ XLI).

63 «Morbi itaque plurimi ab insectis sunt» (§ XLIII).

64 De insectorum utilitate ( $\$$ XLIV-LI).

65 «Ipse rerum Creator omnium, dum cuncta, quae fecerat, vidisset, ea valde bona invenit» $(\S \mathrm{XLV})$.

66 «quod in instrumentum supplicii supremo Numini inservire possint, \& saepe inservierint» (a.a.O.).

67 «Medicamenta nobis praebeant» (§ XLVI). 
spezifische Antidote» ${ }^{68}$. Im folgenden $\S$ XLVIII $^{69}$ wird diese These mit Beispielen für heilsame Wirkungen beinahe aller «Insekten» vom Wurm über die Spinnen bis hin zu den Skorpionen untermauert; als Fazit schreibt Quarin: «Vielleicht gibt es kein Insekt, dass einen medizinischen Nutzen entbehrt; freilich hat manchmal unsere Kunst noch nicht jenen Grad der Glückseligkeit erlangt, dass wir diesen vollständig erkennen und durchschauen können: vielleicht wird die Nachwelt glücklicher sein und Dinge entdecken, die uns heutzutage noch in tiefer Dunkelheit liegend verborgen bleiben ${ }^{70}$.

Schliesslich widmet er den vorletzten Abschnitt seiner Arbeit dem Nutzen, den Insekten auch als Nahrungsmittel haben können, um abschliessend noch einmal den allgemeinen Nutzen, der aus der Beschäftigung mit Insekten erwachsen kann, zu betonen - mit eindrücklichen Worten, die auch einen tiefen Glauben bezeugen: Kaum irgendwoanders könne ein wissbegieriger Forscher eine solche Vielfalt und so kunstvolle Strukturen finden, und hierin wie auch in der Symmetrie und den Verwandlungen der Insekten werde aufs Herrlichste der göttliche Wille offenbar: «Daher kommt und schaut die Werke Gottes, denkt darüber nach und übt euch darin, sie zu erkennen:Herrlich nämlich sind Deine Werke, o Herr; alles hast Du in Weisheit erschaffen; erfüllt ist die Erde von Deinem Besitz» ${ }^{71}$.

\section{$\mathbf{V}$}

Joseph Quarins Arbeit ist - wie diese kurze Wiedergabe der wesentlichen Inhalte zeigt - nicht originell oder gar bahnbrechend. Es ist keine Arbeit, die die Insektenkunde durch neue, auf eigener Anschauung beruhende Erkenntnisse weitergebracht und den Fortgang der wissenschaftlichen Entomologie beschleunigt hat ${ }^{72}$.

68 «imo vero antidota inde possidemus» ( $\$$ XLVII).

69 Dieser Abschnitt ist mit über drei Seiten bei weitem der umfassendste.

70 «Forte nullum insectum est, quod utilitate medica caret; licet nondum eum felicitatis gradum ars nostra attigerit, ut omnem hanc cognitam ac perspectam possideamus: felicior forsan posteritas plura deteget, quae hodierna die profundis tenebris involuta adhuc nos latent» (§ XLIX).

71 «Venite igitur, \& videte opera Dei, meditemini in illis, \& in adinventionibus ejus exerceamini: magnificata enim sunt opera Tua Domine; omnia in sapientia fecisti: impleta est terra possessione Tua» ( $\$ \mathrm{LI})$.

72 Dies war jedoch, daran sei hier noch einmal erinnert, auch ausdrücklich nicht das Ziel des Autors. Ohnehin war Quarin - wie sein weiterer Lebensweg und seine weiteren Veröffentlichungen zumindest vermuten lassen - nicht sonderlich an der Forschung und wahrscheinlich noch weniger an der Insektenkunde als theoretischer Wissenschaft interessiert: Er war vielmehr ein Praktiker, dessen Interesse weniger der Forschung als der Anwendbarkeit und Anwendung der Forschungsergebnisse galt. 
Gerade in diesem Mangel an Originalität liegt jedoch der Reiz für den heutigen Leser. Gerade weil Quarin nicht eigene Ergebnisse präsentiert, sondern lediglich zusammenfasst, was ein begabter Student zu seiner Zeit an entomologischem Wissen erwerben konnte, bietet seine Arbeit einen guten Überblick über den Stand der wissenschaftlichen Entomologie in der Mitte des 18. Jahrhunderts.

Die präzisen Quellenangaben des Studenten Quarin eröffnen darüber hinaus die Möglichkeit, neben dem Stand der Wissenschaft die historischen Entwicklungslinien der Entomologie von der Antike bis zu seiner Zeit nachzuzeichnen. Und genau diese Kombination ist es, die Quarins Promotionsarbeit noch heute als wichtiges Dokument erscheinen lässt.

\section{Literatur:}

Beier, Max:The early naturalists and anatomists during the renaissance and seventeenth century. In:Smith, Ray/Mittler, Thomas/Smith, Carroll (Hrsg.): History of entomology.Palo Alto 1973, S. 81-94.

G (?): Quarin, Joseph. In: Hirsch, August (Hrsg.): Biographisches Lexikon der hervorragenden Ärzte aller Zeiten und Völker. 2. Auflage, Berlin/Wien 1929-1935, Band IV, S. 696-7.

Gessner, Fritz (Hrsg.): Handbuch der Biologie. Band VI/1. Konstanz 1962.

Jahn, Ilse/Löther, Rolf/Senglaub, Konrad: Geschichte der Biologie - Theorien, Methoden, Institutionen und Kurzbiographien. 2. Auflage, Jena 1985.

Lesky, Erna: Gerard van Swieten. Auftrag und Erfüllung. In: Lesky, Erna/Wandruzka, Adam (Hrsg.): Gerard van Swieten und seine Zeit. Wien/Köln/Graz 1973, S. 11-62.

Morge, Günter: Entomology in the Western world in antiquity and in medieval times. In: Smith, Ray/Mittler, Thomas/Smith, Carroll (Hrsg.): History of entomology. Palo Alto 1973, S. 37-80.

Nauck, Ernst Theodor: Die Doktorpromotionen der medizinischen Fakultät Freiburg im Breisgau. Freiburg 1958.

Puschmann, Theodor: Die Medizin in Wien während der letzten hundert Jahre. Wien 1884.

von Quarin, Joseph: Entoma noxia et utilia physico-medice considerata. Freiburg 1751.

Schaub, Friedrich (Hrsg.): Die Matrikel der Universität Freiburg i. Br. von 1656-1806. Band I: Text und Anmerkungen. Freiburg 1955.

Schreiber, Heinrich: Geschichte der Albert-Ludwigs-Universität zu Freiburg im Breisgau III. Theil. Freiburg 1859.

Seidler, Eduard: Die medizinische Fakultät der Albert-Ludwigs-Universität Freiburg im Breisgau - Grundlagen und Entwicklungen. Berlin/Heidelberg 1991.

Toellner, Richard: Hermann Boerhaave (1668-1738) In: v. Engelhardt, Dietrich / Hartmann, Fritz (Hrsg.): Klassiker der Medizin. München 1991, Band I, S. 215-230.

Tuxen, S.L.: Entomology systematizes and describes. In: Smith, Ray/Mittler, Thomas/Smith, Carroll (Hrsg.): History of entomology. Palo Alto 1973, S. 95-118.

Vogel, Günter/Angermann, Hartmut: dtv-Atlas zur Biologie - Tafeln und Texte. 2 Bände, München 1967.

Welcker, Carl: Josef Freiherrn von Quarin's Leben und Wirken - Eine Festrede. Ausserordentliche Beilage zu Nr. 50 des XVI. Jahrgangs der Österreich. Zeitschrift f. prakt. Heilkunde. Wien 1870 [= Österreich. Zeitschrift f. prakt. Heilkunde 16 (1870) S. 847-850].

Zeeden, Ernst Walter: Die Freiburger Universität von der theresianischen Zeit bis zum Übergang an Baden. In: Müller, Wolfgang (Hrsg.): Freiburg in der Neuzeit. Bühl 1972, S. 48-68. 